\title{
Based on network pharmacology to explore the molecular mechanisms of astragalus membranaceus for treating T2 diabetes mellitus
}

\author{
Jie $\mathrm{Li}^{1}$, Yanqin Huang ${ }^{2}$, Sen $\mathrm{Zhao}^{3}$, Qiuyue Guo ${ }^{1}$, Jie Zhou ${ }^{1}$, Wenjing Han ${ }^{1}$, Yunsheng Xu ${ }^{4}$ \\ ${ }^{1}$ College of First Clinical Medicine, Shandong University of Traditional Chinese Medicine, Jinan 250355, China; ${ }^{2}$ Department of Endocrine, \\ Affiliated Hospital of Shandong University of Traditional Chinese Medicine, Jinan 250011, China; Department of Chinese Medicine, The General \\ Hospital of the People's Liberation Army, Beijing 100853, China; ${ }^{4}$ Department of Endocrine, Second Affiliated Hospital of Shandong University of \\ Traditional Chinese Medicine, Jinan 250001, China \\ Contributions: (I) Conception and design: Y Xu, Y Huang, J Li; (II) Administrative support: Y Xu, Y Huang; (III) Provision of study materials or \\ patients: J Li, S Zhao; (IV) Collection and assembly of data: Q Guo, J Zhou; (V) Data analysis and interpretation: J Li, W Han; (VI) Manuscript \\ writing: All authors; (VII) Final approval of manuscript: All authors. \\ Correspondence to: Yunsheng Xu. Department of Endocrine, Second Affiliated Hospital of Shandong University of Traditional Chinese Medicine, \\ Jinan 250001, China. Email: xysnfm65@163.com.
}

Background: Astragalus membranaceus refers to a type of traditional Chinese medicine (TCM) used to treat type 2 diabetes mellitus (T2DM), whereas its molecular mechanism remains unclear. In the presented study, network pharmacology was performed to analyze the molecular mechanism of astragalus membranaceus against T2DM.

Methods: First, we found common targets of astragalus membranaceus and disease, protein-protein interaction (PPI) network was built by String, and then key targets were screened from these common targets by topological analysis. Subsequently, common targets were introduced into DAVID to achieve the results of gene ontology (GO) and KEGG enrichment analysis. The therapeutic effect of astragalus was observed, and several key targets were verified by an animal experiment.

Results: First, 13 key targets (EGFR, KDR, SRC, ERBB2, FYN, ESR1, AR, HSP90AA1, PTGS2, ABCG2, AB1, MMP2, and CYP1) were found by topological analysis. Then, the results of GO and KEGG suggested that the anti-diabetes effect of astragalus membranaceus was strongly associated with the activation of receptor protein tyrosine kinase (RPTK). The results of animal experiments revealed that astragalus could enhance the morphology of rat pancreas and up-regulate the expression of tyrosine receptor.

Conclusions: In brief, 13 key targets were found in this study, and astragalus membranaceus was found upregulating insulin signaling pathways by improving the activity of casein kinase, regulating lipid metabolism, and enhancing insulin resistance to treat T2DM. The present study lays a basis for subsequent experimental research and broadens the clinical application of astragalus membranaceus.

Keywords: Network pharmacology; molecular mechanisms; astragalus membranaceus; type 2 diabetes mellitus (T2DM)

Submitted Oct 08, 2019. Accepted for publication Oct 14, 2019.

doi: $10.21037 /$ atm.2019.10.118

View this article at: http://dx.doi.org/10.21037/atm.2019.10.118 


\section{Introduction}

Type 2 diabetes mellitus (T2DM) refers to chronic metabolic disease for the interactions between genetic and environmental factors. The rapidly rising incidence of T2DM is imposing a major threat to global public health. According to the World Health Organization estimated the number of T2DM patients would increase to 642 million by 2040, which will impose a heavy financial burden on society and families (1). Currently, medicines used clinically to treat DM include insulin, insulin sensitizers, and insulin-secreting drugs, whereas their treatment has numerous side effects [e.g., hypoglycemia (2), gastrointestinal discomfort (3), and shortage of preventing the complication effectively (3)]. Given this, many doctors and patients have begun to seek alternative medicines to treat DM. Herbal medicine, as a type of alternative drug, displays the merits of low sideeffects and less irritation to the gastrointestinal tract. It has been demonstrated to have a good clinical effect on the treatment of T2DM (4-6). Astragalus membranaceus is a familiar and vital Chinese medicine with well-defined phytochemicals, and it has long been adopted extensively and shown explicit clinical treatments regarding antidiabetes, whereas the molecular mechanism has not been clearly elucidated. Thus, studying the active components, targets, and mechanisms of astragalus membranaceus helps explain its molecular mechanisms and broaden its clinical application.

Traditional Chinese medicine (TCM) treats diseases in a holistic manner, while TCM herb and recipes are multi-component, multi-target agents at the molecular level, making its pharmacodynamic component and molecular mechanism not certain. Thus, it is necessary to develop a new way to explain molecular mechanisms and pharmacological effects. As fueled by the advancement of network technique and bioinformatics, the network pharmacology has been confirmed as a feasible choice to explicate the material composition and molecular mechanism of TCM effectively and systemically $(7,8)$. In 2007, Hopkins proposed the concept of network pharmacology (9). The complex network of drug ingredients, targets, and diseases were built, and the mechanism of drug and compounds action was discussed based on the analysis of the intervention of drug and compounds in the disease network. Besides, network pharmacology seeks critical targets by building complex networks at distinct levels and assesses the molecular mechanisms of drug treatment. Through network pharmacology, Li (10) found that tumor necrosis factor (TNF) was the critical target of salvia miltiorrhiza against bone marrow fibrosis. He also reported that cryptotanshinone of salvia miltiorrhiza might treat molecular function (MF) by mediating the JAK-STAT signaling pathway and transforming growth factor (TGF) signaling pathway. $\mathrm{Bi}$ (11) explored the key targets and the anti-tumor mechanism of calycosin against colorectal cancer by using system pharmacology approach successfully.

In the present study, the active components of astragalus membranaceus were identified by TCMSP database, and the ingredient-targets were predicted by Swiss TargetPrediction database. Subsequently, the disease-targets were found from DisGeNET database and Genecards database. Besides, common targets for herb and disease were given intersection and introduced into String for protein-protein interaction (PPI) analysis, and Cytoscape was employed to visualize the results of PPI. Based on the topological analysis, some of the critical targets were selected from the common targets as the key targets of astragalus for the treatment of T2DM. Also, gene ontology (GO) and KEGG enrichment of common targets was analyzed, and the therapeutic effect of astragalus and several critical targets was verified by experiments. The workflow is displayed in Figure 1.

\section{Methods}

\section{Screening the chemical components of astragalus membranaceus and predicting the component-targets}

The chemical ingredients of astragalus membranaceus were screened from TCMSP (http://1sp.nwu.edu.cn/ tcmsp.php). TCM is mostly oral preparations, i.e., being digested, distributed, metabolized, and excreted to target organs and tissues, which is known as the ADME process. TCMSP database details the ADME parameters of each component [e.g., oral bioavailability (OB), druglikeness (DL), Caco-2 cell permeability, half-life, bloodbrain barrier, as well as fat-water distribution coefficient]. Ingredients meeting the demands of both $\mathrm{OB} \geq 30 \%$ and DL $\geq 0.18$ were selected to find the effective components of astragalus membranaceus $(12,13)$. OB represents the oral availability of pharmaceutical ingredients, and DL refers to the similarity between a component and a known drug, which can act as an indicator of its proximity to a listed drug (12). Subsequently, 20 components in astragalus membranaceus were selected (Table 1). An ingredient 


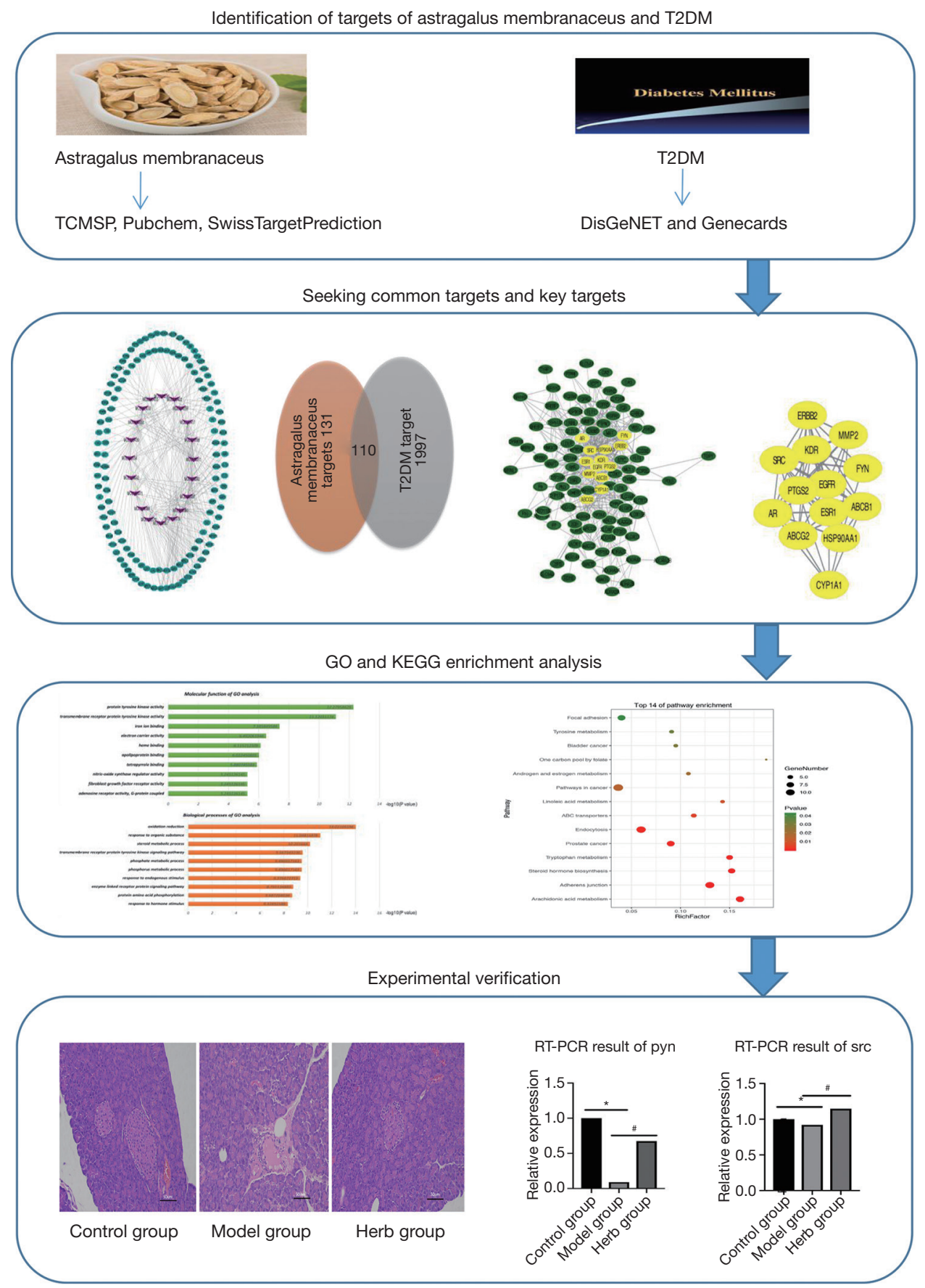

Figure 1 Workflow for exploring the molecular mechanisms of astragalus membranaceus against T2DM. T2DM, type 2 diabetes mellitus; GO, gene ontology; RT-PCR, reverse transcription-polymerase chain reaction.

(MOL000374) was removed because it does not have the canonical SMILE string and 3D structures to predict the component-targets. Moreover, astragaloside IV was included in this study because according to many studies, it had a good anti-diabetes effect, though it does not meet the inclusion criteria $(14,15)$. Lastly, 20 active ingredients were taken for subsequent study. Next, 20 active components were inputted into PubChem (https://pubchem.ncbi.nlm. 
Table 1 Twenty active components of astragalus membranaceus that meeting the demands of both OB $\geq 30 \%$ and DL $\geq 0.18$ were obtained from TCMSP

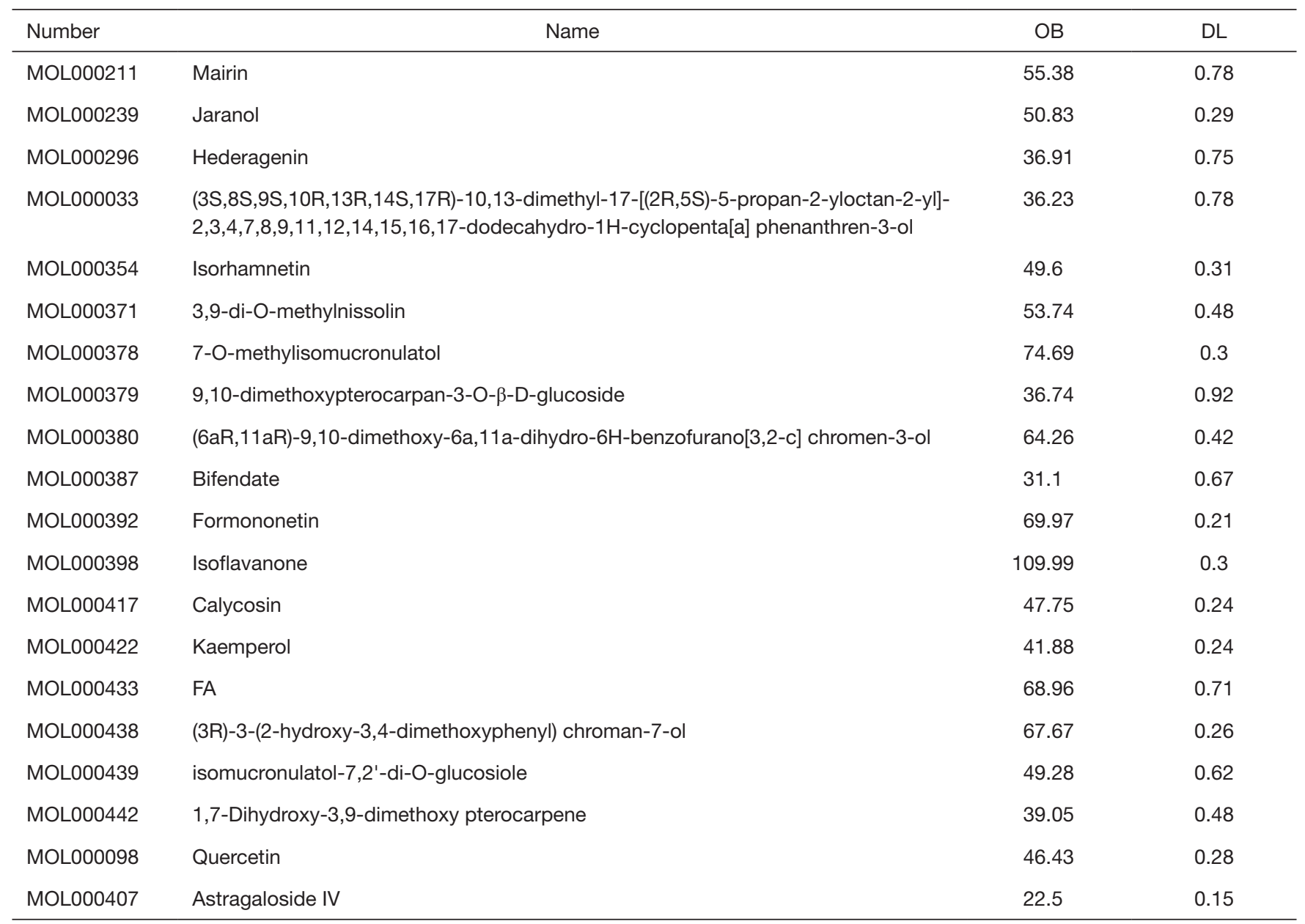

OB, oral bioavailability; DL, drug-likeness; FA, folinic acid.

nih.gov/) to achieve the canonical SMILES strings, and the resulting strings were sent to Swiss TargetPrediction (http:// www.swisstargetprediction.ch) to yield the targets of each ingredient. In the study, the species was set as "human", and the prediction results of the target of the platform were collected and then classified.

\section{Seeking out disease-related targets}

With "diabetes mellitus, type 2" as the keywords, DisGeNET database(http://www.disgenet.org) and Genecards (https://www.genecards.org) were used to search and screen the known disease-targets for the subsequent study, and the repeated targets in the search results were discarded. DisGeNET database integrates gene-disease associations (GDAs) of multiple databases and considerable literature, and it adopts text mining technology to analyze the correlation between Mendelian diseases, complex diseases, and environmental diseases (16). UniProtKB (http://www.uniprot.org/) was used to get the standard targets' names with the organism selected as "homo sapiens".

\section{Searching for common targets and key targets of astragalus membranaceus and T2DM}

The common targets of drug and disease were found, and a Venn diagram was drawn (Figure 2). Next, the common targets were inputted into String (http://string-db.org/, version 10.5) for PPI analysis, and Cytoscape 3.6.1 (http:// 


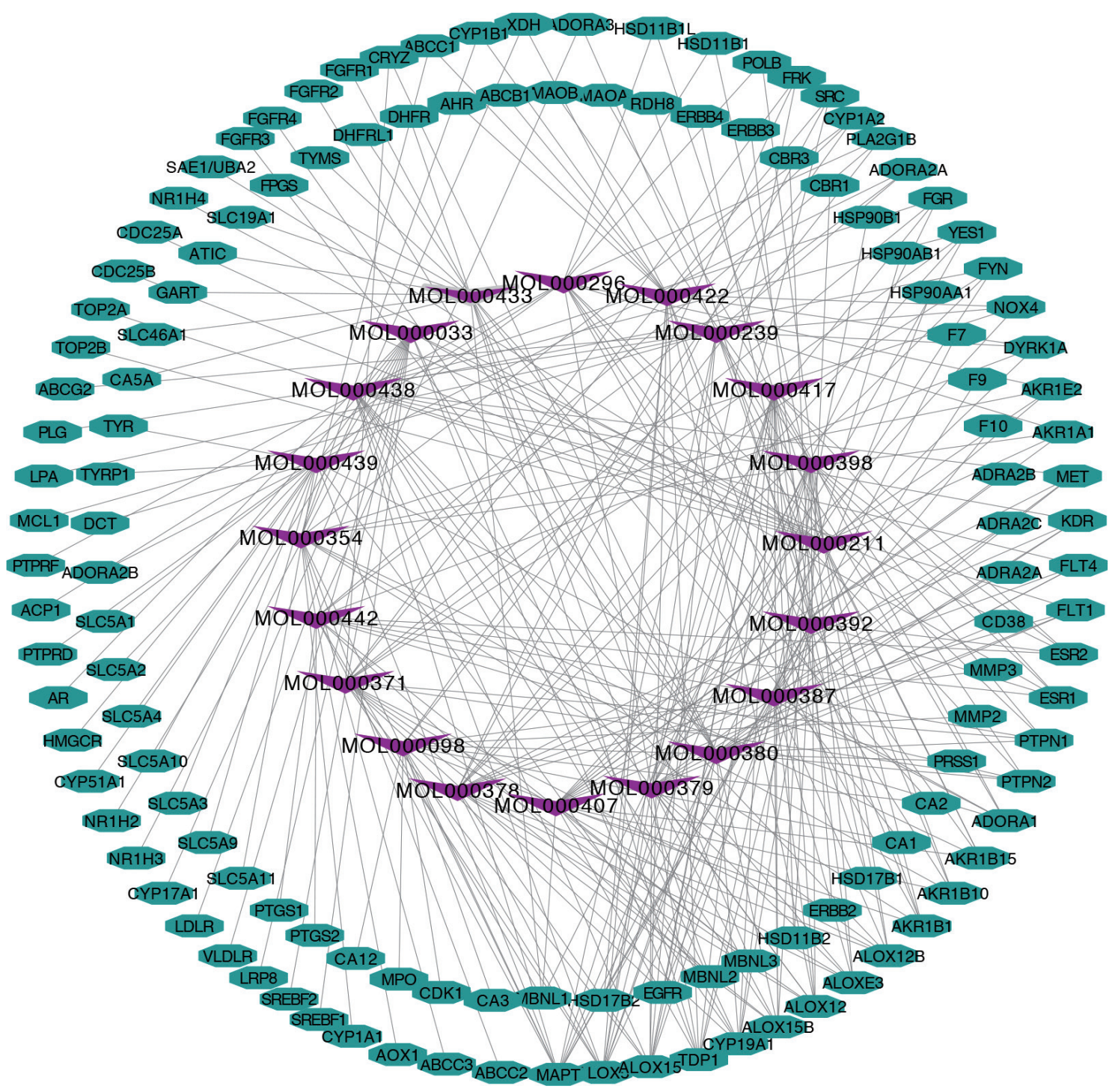

Figure 2 Component-component target network (purple triangles represent components contained in astragalus membranaceus, and blue circles represent component targets).

www.cytoscape.org/)was adopted to visualize the results. The String database refers to a biological database for prediction of PPIs, with data from several sources (e.g., experimental data, computational prediction methods, and a public text set) (17). Next, the protein interaction relationship was obtained, and the results were exported in tab-separated value (TSV) format. String integrates known and estimated PPIs and then assesses PPI with confidence score ranges (low confidence: score $<0.4$; medium: $0.4-0.7$; high: $>0.7$; highest: $>0.9)$ (18). In this study, PPIs with medium confidence scores $(>0.4)$ were gained for further exploration.
The topological analysis covers degree centrality (DC), betweenness centrality (BC), closeness centrality (CC), eigenvector centrality (EC), and network centrality (NC) and local average connectivity (LAC) (19). DC is a vital parameter to measure the local centrality of a node. A node with high DC score can be the central node of the network. It often engages in essential life activities, executes a key biological function, and plays an irreplaceable role to maintain the stability of the interaction network, making it a research hotspot for drug targets (20). BC refers to the number of times a node acts as the shortest bridge between 
the other two nodes. The higher a node mediates, the more centrality it has. In this experiment, the nodes with DC score and BC score greater than 2 times the median were taken as important nodes in the network, namely "key targets". We assumed that they were the critical targets for anti-diabetes in astragalus. In this study, the median of DC was 4 , and the median of BC was 0.00558038 .

\section{GO and pathway enrichment analysis}

The 110 common targets were adopted for GO and KEGG enrichment analysis by DAVID database (https://david. ncifcrf.gov/, ver. 6.8). DAVID refers to a bioinformatics database that provides systematic and comprehensive bioinformatics annotations for large-scale gene or protein lists based on both biological data and analysis tools (21). GO analysis is a system extensively used for gene function classification in the field of biology, which is primarily adopted to express the function of gene products, including cell function, MF and biological process (BP). Furthermore, 110 targets were uploaded to the DAVID for KEGG pathway enrichment analysis. The GO terms and pathway terms with $\mathrm{P}$ value $<0.05$ were taken for subsequent study.

\section{Animal experiments verified the therapeutic effects of astragalus and randomized two key targets}

\section{Model preparation, grouping, and dissection of rats}

Twenty-four male Wistar rats with the bodyweight of $130 \pm 10 \mathrm{~g}$ was purchased from Beijing Vital River Laboratory Animal Technology Co., Ltd. (Beijing, China). This study was approved by the Animal Ethics Committee of Affiliated Hospital of Shandong University of Traditional Chinese Medicine and conducted following the principles of the Care and Use of Laboratory Animal (Ethical approval number: AWE-2-19-001). After acclimatization for one week, animals were randomly split into three groups (8 rats/group): the control group, the model group, as well as the herb group. The normal rats were fed with common pellet diets, while other groups were fed by a high fat diet (67\% maintenance feed $+10.0 \%$ lard $+20.0 \%$ sucrose $+2.5 \%$ sodium cholate and $2.5 \%$ cholesterol). After 28 days of dietary intervention, a dose of $35 \mathrm{mg} / \mathrm{kg}$ STZ (Sigma, \#18883-66-4, USA) was administrated to the model group and astragalus membranaceus group. Three days later, rats were supposed to be T2DM model when their FBG levels reached over $16.7 \mathrm{mmol} / \mathrm{L}$. The normal group and the model group were given distilled water per day by gavage, and herb group was given $(5.53 \mathrm{~g} / \mathrm{kg} / \mathrm{d})$ astragalus membranaceus (purchased by affiliated hospital of Shandong University of Traditional Chinese Medicine, China) water decoction, 28 days, the pancreas tissue was dissected for the subsequent study.

\section{The morphology of rat pancreas was observed by} hematoxylin and eosin (HE) staining

After dissection, each pancreas tissue was fixed in 4\% (w/v) paraformaldehyde over $24 \mathrm{~h}$. Dehydrated with graded ethanol and embedded in paraffin. Subsequently, $4 \mathrm{~mm}$ sections were rehydrated and then stained with HE. The program of staining was obtained under an IX51 microscope (Olympus Corporation, Tokyo, Japan).

\section{Several key targets were randomly verified by quantitative reverse transcription-polymerase chain reaction (qRT-PCR)}

First, the designed primers were fabricated, and the mRNA sequence of the rat was searched in the (NCBI) database. The primers were designed using primer 5 biological software. After primers design, they were chemically synthesized by Shanghai Biotech Co., Ltd., and the specific procedure was followed in line with the instructions. Each primer sequence is: FYN F: 5'-ATTGGCCCGGATTGA AG-3'; FYN R: 5'-TGAGCTCGTGCAGGGAGATC-3'; SRC F: 5'-CGGGATCCCGATGGGCAG- CAACAAGA GC-3'; SRC R: 5'-CGGAATTCCGCTATAGGT-TCTC CCCGGGC-3'; GAPDH F: 5'-CCCACGGCAAGTTCA ACGG-3'; GAPDH R: 5'-CTTTCAGAGGGGCCATC CA-3'.

First, an appropriate amount of pancreatic tissue was ground and cleaved, RNA was extracted by Total RNA Kit (Tiangen, China), and then RNA concentration was ascertained by absorbance. Using FastQuant RT Kit (Tiangen, China), $10 \mu \mathrm{L}$ of gDNA removal reaction system and $10 \mu \mathrm{L}$ of reverse transcription reaction system were separately configured, and the two systems were mixed at $42{ }^{\circ} \mathrm{C}$ for $15 \mathrm{~min}$, incubated at $95^{\circ} \mathrm{C}$ for $3 \mathrm{~min}$, and then placed on ice to obtain cDNA to be used in subsequent experiments. Establishment of a $20 \mu \mathrm{L}$ reaction system containing cDNA, forward and reverse primers, $2 \times$ Super real Premix Plus, and Rnase-free $\mathrm{ddH} 2 \mathrm{O}$ was put into Roche 480 fluorescent polymerase chain analyzer for qRTPCR reaction, $95{ }^{\circ} \mathrm{C} 15$ min pre-denaturation, $95{ }^{\circ} \mathrm{C} 10 \mathrm{~s}$ denaturation, $55^{\circ} \mathrm{C} 20 \mathrm{~s}$ annealing, $72{ }^{\circ} \mathrm{C} 20 \mathrm{~s}$ extension, a total of 40 cycles. The data were calculated and analyzed based on the $2^{-\Delta \Delta c t}$ method. 


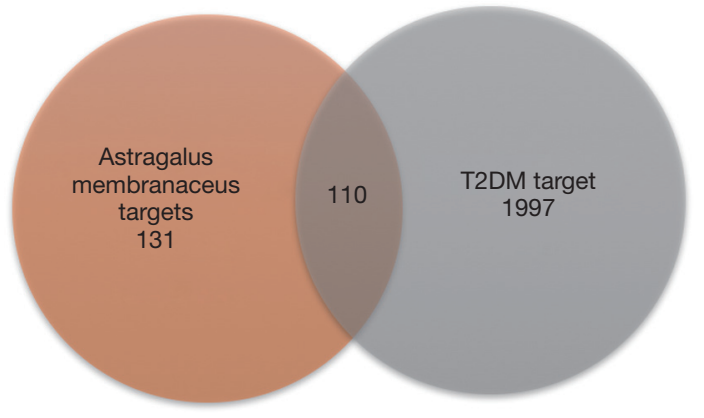

Figure 3 The intersection of astragalus membranaceus and T2DM targets, with a total of 110 targets. T2DM, type 2 diabetes mellitus.

\section{Results}

\section{Identification of targets of astragalus membranaceus and T2DM in various databases}

A total of 87 ingredients from astragalus membranaceus were picked from the TCMSP database, and effective components were estimated using DL and $\mathrm{OB}$ (OB $\geq 30 \%$, $\mathrm{DL} \geq 0.18$ ). 20 active ingredients (Table 1) were lastly chosen for further investigation. One hundred and thirtyone component-targets were identified from 20 active ingredients of this herb using SwissTargetPrediction database to delete the repetitive targets. Componentcomponent targets network was built to elucidate intuitive interactions in astragalus membranaceus (Figure 2). There were 151 nodes and 300 edges in the network. Most component nodes related to multiple target nodes such as so flavanone, hederagenin, and calycosin, etc. The network suggested that many component-targets could be adjusted by multiple ingredients (e.g., SRC, EGFR, CYP19A1), which may be vital component-targets in astragalus membranaceus against T2DM, revealing that the procedure of astragalus membranaceus in the treatment of DM has the characteristics of multiple components and multiple targets. According to DC, the top three active ingredients were (3R)-3-(2-hydroxy-3,4-dimethoxyphenyl) chroman7-ol, isoflavone, and calycosin, with a score of 15, 13 and 13, respectively. Besides, we adopted DisGeNET database and Genecards database to find 1997 disease targets with no repeats were collected for additional research.

\section{Seeking common targets and key targets and built PPI networks separately}

The disease-targets and the drug-targets were intersected to find 110 common targets, which were important targets for the treatment of the disease, and the results were shown by Wayne diagram (Figure 3). The mentioned 110 targets were inputted into the STRING database to build a PPI network (Figure 4). There were 106 points and 546 edges in the network. According to the twice median of BC and DC, 13 critical nodes, namely "key targets", were further screened. They were primarily tyrosine kinases, sex hormones, including EGFR, KDR, SRC, ERBB2, FYN, ESR1, AR, HSP90AA1, PTGS2, ABCG2, AB1, MMP2, CYP1 (Figure 5). These 13 targets can be used as valuable targets for astragalus membranaceus in the treatment of T2DM and deserve subsequent study.

\section{Enrichment analysis by GO and KEGG}

According to $\mathrm{P}$ value $<0.05$, the top ten items of MF of GO enrichment were protein tyrosine kinase activity, transmembrane receptor protein tyrosine kinase (RPTK) activity, iron ion binding, electron carrier activity, heme binding, apolipoprotein binding, tetrapyrrole binding, nitricoxide synthase regulator activity, adenosine receptor activity, G-protein coupled, nitric-oxide synthase regulator activity, ligand-dependent nuclear receptor activity, protein kinase activity, lipoxygenase activity. And the top ten items of BP of GO analysis were oxidation-reduction, response to organic substance, steroid metabolic process, transmembrane RPTK signaling pathway, phosphorus metabolic process, phosphate metabolic process, response to endogenous stimulus, enzyme-linked receptor protein signaling pathway, protein amino acid phosphorylation, response to hormone stimulus (Figure 6). The results of the GO analysis showed that astragalus treatment of diabetes was strongly associated with the activation of tyrosine kinase.

According to the $\mathrm{P}$ value, a total of 14 pathways were screened by KEGG analysis, involving lipid metabolism, tyrosine metabolism, angiogenesis, insulin resistance and other aspects, including arachidonic acid metabolism, tyrosine metabolism, adherens junction, steroid hormone biosynthesis, tryptophan metabolism, prostate cancer, endocytosis, $\mathrm{ABC}$ transporters, linoleic acid metabolism, pathways in cancer, androgen and estrogen metabolism, one carbon pool by folate, bladder cancer, focal adhesion (Figure 7).

\section{HE staining results}

The results (Figure 8) revealed that the islets of the control 


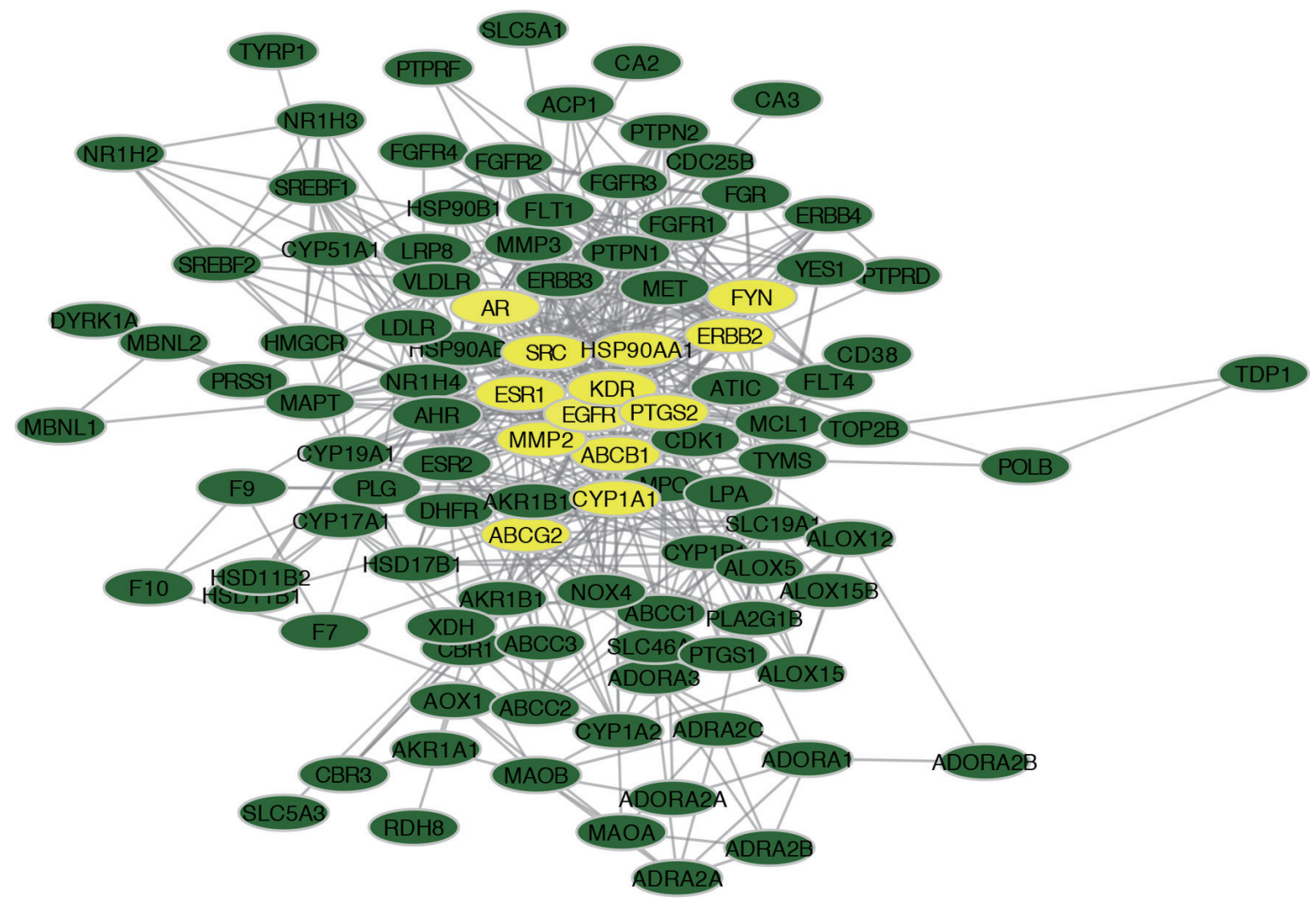

Figure 4 PPI network of 110 common targets (astragalus membranaceus and T2DM). According to topological analysis, 13 yellow targets are key nodes. T2DM, type 2 diabetes mellitus; PPI, protein-protein interaction.

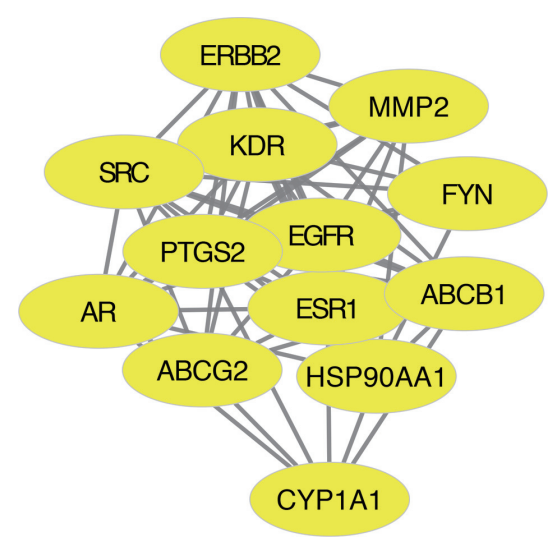

Figure 5 PPI network between 13 key targets. PPI, proteinprotein interaction.

group were in the center of the exocrine part of the pancreas, with a regular shape and a clear outline. The island is rich in capillaries, with considerable islet cells and clear boundaries with exocrine glands. In the model group, the islet shape was irregular, the islet atrophy was obvious, the contour was less rounded, the edge was unclear, and the number of islet cells in the island significantly decreased. These findings suggested that type 2 diabetic rats were successfully modeled. After treatment with astragalus membranaceus, the structure of pancreatic islet cells was significantly strengthened, and the arrangement was orderly, close to the morphological characteristics of normal tissues. Moreover, there were new island cells and abundant capillaries on the island. It was therefore implied that there is a favorable effect after Chinese medicine treatment.

\section{The qRT-PCR results}

The ultraviolet spectrophotometer was adopted to detect the total RNA extracted, A260/A280 score were 1.8-2.1 and close to 2.0, indicating that the total RNA extracted was DNA-free, high purity and good integrity, which could be used for subsequent experiments. The results showed that, compared with the control group, PYN and SRC 


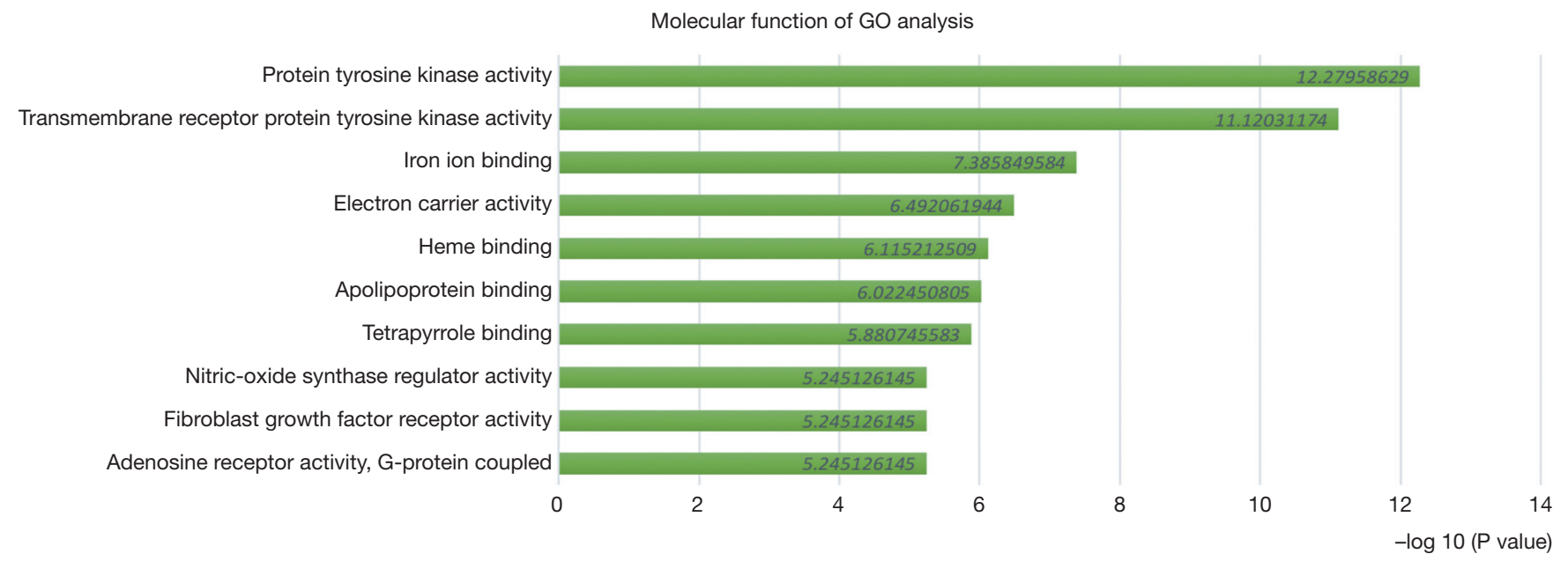

Biological processes of GO analysis

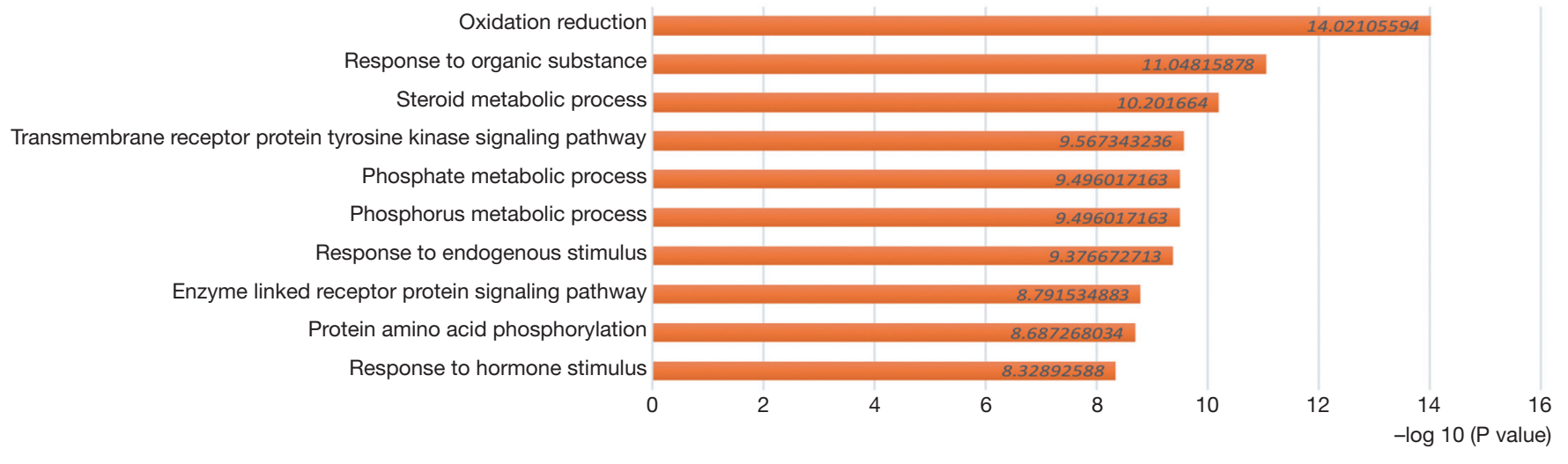

Figure 6 GO analysis of 110-common targets (astragalus membranaceus and T2DM). T2DM, type 2 diabetes mellitus; GO, gene ontology.

expressions were reduced in the model group, and this herb could improve PYN and SRC expressions (Figure 9).

\section{Discussion}

The global incidence of diabetes is extremely high, bringing heavy mental pressure and financial burden to society and individual families. Medicines utilized include insulin sensitizers, insulin-secreting drugs, and insulin, whereas they have numerous side effects. Accordingly, the search for complementary and alternative medicine has become a top priority. As a traditional Chinese herb, astragalus membranaceus has been broadly selected in the treatment of DM in clinical practice. In the present study, network pharmacology was used to explore the material basis and molecular mechanism of astragalus membranaceus for treatment of T2DM.

First, the active ingredient and target of this TCM were found, and a network of Chinese medicine ingredients and drug targets was built. According to topological analysis, the critical compounds for treating diabetes were (3R)-3-(2hydroxy-3,4-dimethoxyphenyl) chroman-7-ol, isoflavanone, and calycosin, with a score of 15,13 and 13, respectively, which are all flavonoids. Studies on the mechanism of action suggested that flavonoids can improve the metabolism of sugar and lipid, enhance insulin resistance, inhibit the activity of relevant glucose metabolic enzymes, and avoid oxidative damage of DM while delaying the occurrence and the development of diabetic complications $(22,23)$. Accordingly, the flavonoids of TCM may be a novel antidiabetic drug, showing bright development prospects. We speculated that these three compounds might be the key compounds in the treatment of T2DM with astragalus membranaceus.

One hundred and ten common targets were found for drug and diseases, which might be targets for astragalus 


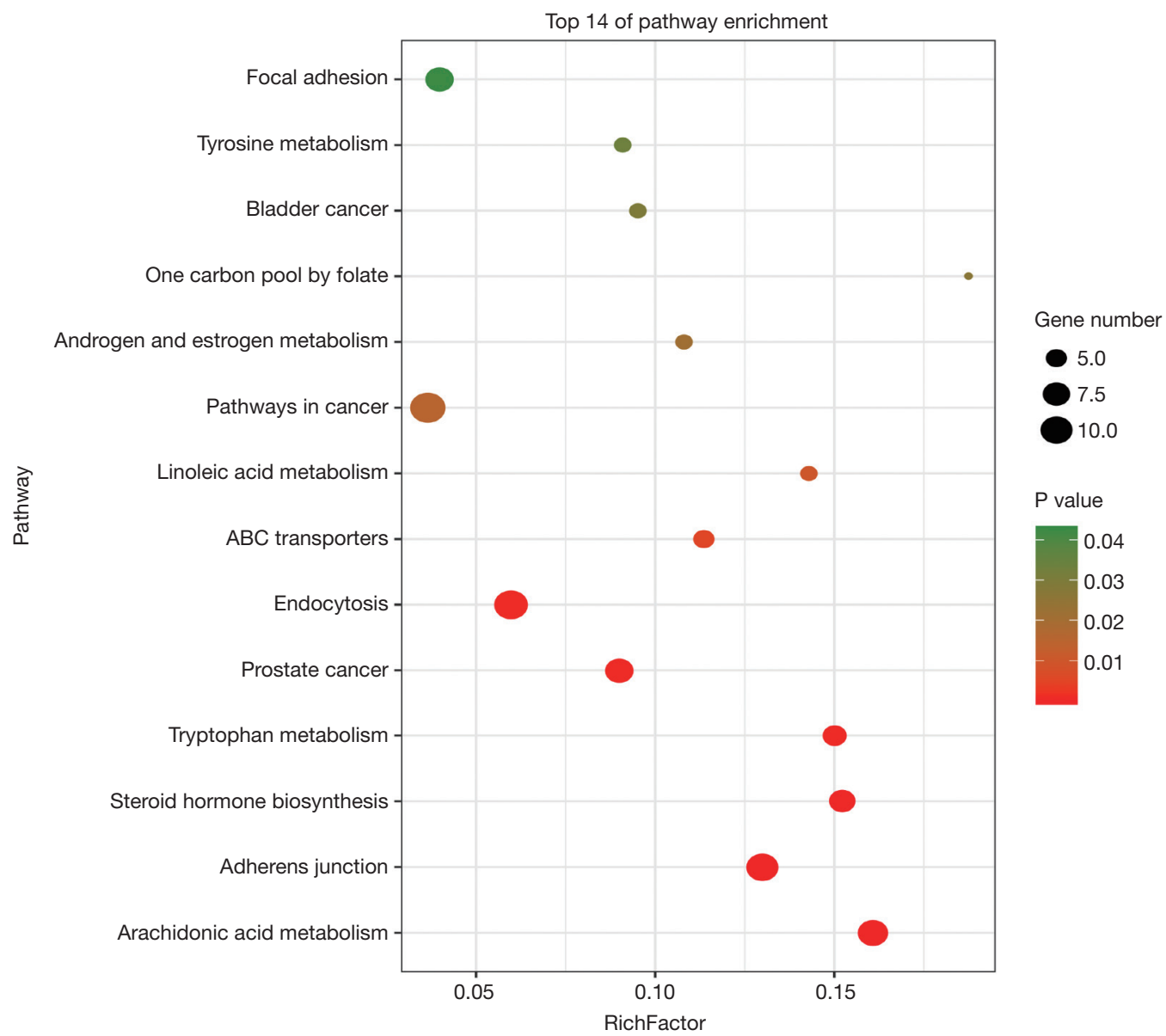

Figure 7 KEGG analysis of 110-common targets (the vertical axis represents the pathway name; the horizontal axis represents the Rich factor).

membranaceus to play an anti-diabetic role. Based on the topological analysis, we further found the 13 critical targets from the 110 common targets for subsequent study. The 13 hub targets primarily include RPTK and sex hormones. We considered that astragalus could enhance the activity of tyrosine kinase to enhance insulin signaling, improve insulin resistance, and participate in the treatment of diabetes and complications.

Receptor tyrosine kinase (RPTKs) includes epidermal growth factor receptors (EGFRs) family, vascular endothelial growth factor receptor (VEGFR) family, and insulin-like growth factor 1 receptors (IGF1Rs) family, insulin receptor (INSR) family, platelet-derived growth factor receptors (PDGFRs) family, macrophage colonystimulating factor receptors (MCSFRs) family, nerve growth factor receptor (NGFR) family, and fibroblast growth factor receptor (FGFR) family, and liver cell growth factor receptor (HGFR) family, etc. (24). Tyrosine kinase phosphorylation is vital to insulin signal transduction, and it promotes insulin secretion by promoting insulin signal transduction, enhancing insulin sensitivity, and maintaining blood glucose balance $(25,26)$.

The key nodes screened in this study included EGFR, KDR (VEGFR), SRC, FYN, and ERBB2, all receptor tyrosine kinases. EGFR is a $170-\mathrm{kDa}$ transmembrane receptor tyrosine kinase. Studies on developmental biology suggested that the lack of EGFR will lead to impaired pancreatic islet development in mice. Also, if the EGFR signal is weakened, the beta-cell proliferation will be significantly inhibited (27). EGFR is a member of the ErbB receptor family, and the EGFR signaling pathway in beta cells affects its cellular function, which is considered a multistage coupling effect. First, five different tyrosine residues in the receptor intracellular domain were auto- 


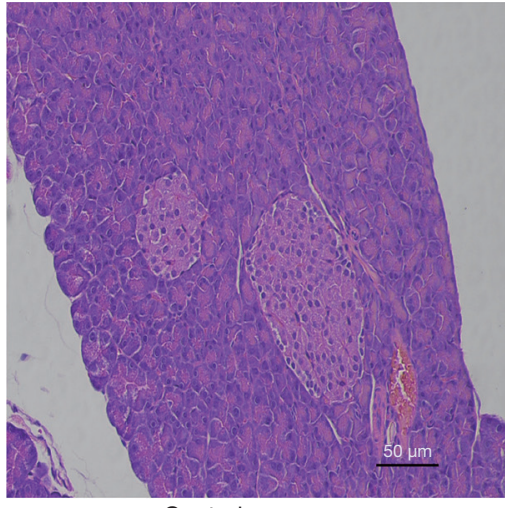

Control group

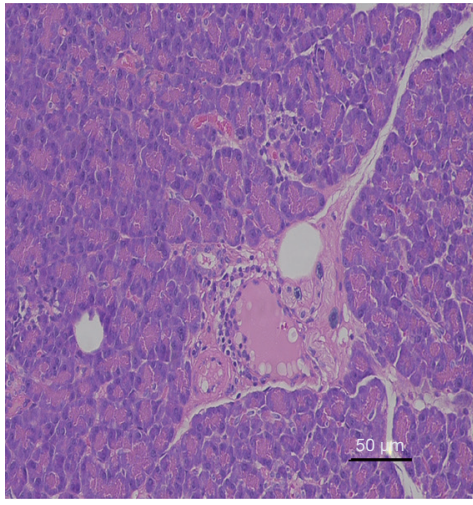

Model group

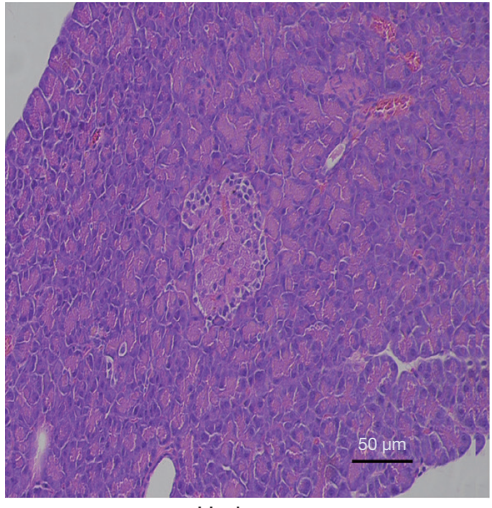

Herb group

Figure $8 \mathrm{HE}$ staining results of rat pancreatic tissue (control group, model group, herb group). HE, hematoxylin and eosin.

RT-PCR result of PYN

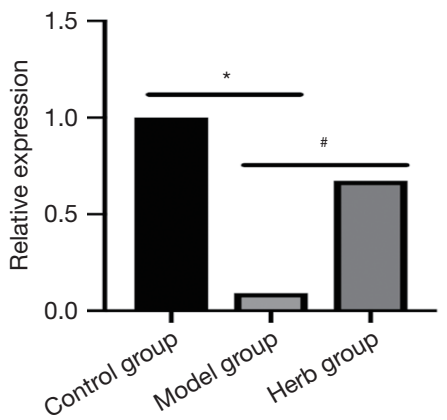

RT-PCR result of SRC

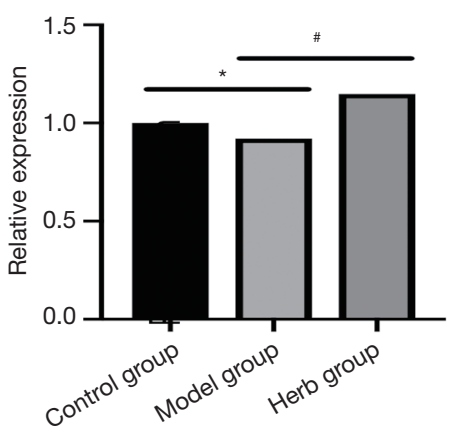

Figure 9 Several key targets were randomly validated by qRT-PCR to verify the reliability of this network pharmacology study. The results of PCR were consistent with those of network pharmacology. *, denotes that $\mathrm{P}$ value $<0.05$ between two group; ${ }^{*}$, denotes that $\mathrm{P}$ value $<0.05$ between two group. qRT-PCR, quantitative reverse transcription-polymerase chain reaction.

phosphorized, activating different phosphorylated tyrosine binding proteins and second messengers. In the meantime, the major downstream signaling pathways of ErbBs (especially EGFR) family include MAPK, PI3k-akt, and cAMP/PKA signaling pathways (28). Studies reported that the relationship between the PI3k-akt pathway and the proliferation of islet cells is closer. Besides, EGFR can affect the pathogenesis of diabetic nephropathy by mediating oxidative stress and endoplasmic reticulum stress (29). On that basis, it was speculated that Astragalus membranaceus could treat both T2DM and diabetic nephropathy by regulating EGFR. HER2/ErbB2, a member of the EGFR family, was associated with insulin resistance. Clinical studies indicated that plasma ErbB2 is significantly positively correlated with glucose, insulin, and HbA1c. When ErbB2 levels were elevated, the incidence of diabetes increased (28).
We speculated that astragalus might also enhance insulin resistance by reducing ErbB2. Three SRC family members have been identified (SRC-1, SRC-2, and SRC-3). The study found that SRC was widely expressed in the body, and the expression of SRC mRNA and protein could be detected in numerous normal tissues and cells (e.g., pancreas, brain, skeletal muscle, heart, lung, liver, kidney, testis, as well as breast) (30). As a co-activator, receptor co-activator SRC1 can activate a variety of nuclear receptors and then induce the expression of key enzymes, e.g., PEPCK and pyruvate carboxylase to regulate sugar metabolism. Some studies had shown that SRC-1 played a key role in regulating fat metabolism, inducing the synthesis of triglyceride (TG) and aggravating hepatic steatosis. SRC-1 knockout mice tend to become obese because of reducing fat consumption and increasing body weight. Accordingly, we speculated that 
astragalus membranaceus could regulate lipid metabolism, improved insulin resistance, and treated diabetes through SRC (31). As a member of the SRC family tyrosine kinases (SFKs), FYN is involved in mediating podocytic injury induced by high glucose, which can be improved by interfering with the expression of FYN, providing a new target for clinical intervention and treatment of diabetic nephropathy (32). KDR (VEGFR) is a tyrosine kinase receptor and a key factor regulating angiogenesis in physiological and pathological conditions. In patients with diabetic retinopathy, the expression of retinal vegfr-2 and VEGF increased (33). Cai et al. found that the massive proliferation of choroid neovascularization in diabetic mice was affected by the activation of hyperglycemic induced VEGF2 signaling pathway (34). Accordingly, we thought that astragalus membranaceus played a therapeutic role in diabetic retinopathy by regulating epidermal growth factor to improve angiogenesis. Sex hormones are strongly associated with diabetes. Estrogen regulates the mechanism of gluconeogenesis by interacting with liver Foxo1 (35). The reduction of estrogen in postmenopausal women accelerates the development of insulin resistance and T2DM. On the other hand, low male androgen levels increase the risk of diabetes (36).

KEGG results showed a total of 14 entries, primarily involving tyrosine, tryptophan metabolism, lipid metabolism, angiogenesis, etc. Moreover, the top ten BP and MF entries in the GO analysis were also associated with tyrosine kinases. These results further verified that the activation of tyrosine kinase, the activation of RPTK signal pathway, and the effect of insulin signal activation act as the critical mechanism of astragalus in the treatment of T2DM.

HE staining results revealed that in the diabetic rat model, astragalus could improve islet morphology, increase beta cells, and treat diabetes. It was fully demonstrated that this TCM is effective in the treatment of T2DM and worthy subsequent studying in the clinic. We randomly selected two hub targets for qRT-PCR validation and confirmed that astragalus did indeed improve tyrosine kinase activity.

In conclusion, this study found 13 key targets and demonstrated that astragalus improves insulin signaling pathways by up-regulating the activity of casein kinase, regulating lipid metabolism, and enhancing insulin resistance to treat T2DM; although further experiments should support this discovery, it contributes an important foundation for subsequent study of the follow-up mechanism and optimization of experimental design.

\section{Acknowledgments}

Funding: This work was supported by the National Natural Science Foundation of China (No. 81673966 and 81603613); Taishan Scholar Engineering Special Funding (No. ts201712097).

\section{Footnote}

Conflicts of Interest: The authors have no conflicts of interest to declare.

Ethical Statement: The authors are responsible for all aspects of the work in ensuring that questions related to the accuracy or integrity of any part of the work are appropriately investigated and resolved. This study was approved by the Animal Ethics Committee of Affiliated Hospital of Shandong University of Traditional Chinese Medicine and conducted following the principles of the Care and Use of Laboratory Animal (Ethical approval number: AWE-2-19-001).

\section{References}

1. Reusch JE Manson JE. Management of type 2 diabetes in 2017: getting to goal. JAMA 2017;317:1015-6.

2. Meneilly GS, Tessier DM. Diabetes, dementia and hypoglycemia. Can J Diabetes 2016;40:73-6.

3. Bonnet F, Scheen A. Understanding and overcoming metformin gastrointestinal intolerance. Diabetes Obes Metab 2017;19:473-81.

4. Chen $X$, Wang H, Jiang $M$, et al. Huangqi (astragalus) decoction ameliorates diabetic nephropathy via IRS1PI3K-GLUT signaling pathway. Am J Transl Res 2018;10:2491-501.

5. Liao H, Hu L, Cheng X, et al. Are the Therapeutic Effects of Huangqi (Astragalus membranaceus) on Diabetic Nephropathy Correlated with Its Regulation of Macrophage iNOS Activity? J Immunol Res 2017;2017:3780572.

6. Park SJ, Nam J, Ahn CW, et al. Anti-diabetic properties of different fractions of Korean red ginseng. J Ethnopharmacol 2019;236:220-30.

7. Luo TT, Lu Y, Yan SK, et al. Network pharmacology in research of Chinese medicine formula: methodology, application and prospective. Chin J Integr Med 2019. [Epub ahead of print].

8. Long S, Yuan C, Wang Y, et al. Network pharmacology 
analysis of damnacanthus indicus C.F.Gaertn in genephenotype. Evid Based Complement Alternat Med 2019;2019:1368371.

9. Zhang R, Zhu X, Bai H, et al. Network pharmacology databases for traditional Chinese medicine: review and assessment. Front Pharmacol 2019;10:123.

10. Li J, Ma X, Liu C, et al. Exploring the mechanism of Danshen against myelofibrosis by network pharmacology and molecular docking. Evid Based Complement Alternat Med 2018;2018:8363295.

11. Bi YH, Zhang LH, Chen SJ, et al. Antitumor mechanisms of curcumae rhizoma based on network pharmacology. Evid Based Complement Alternat Med 2018;2018:4509892.

12. Ru J, Li P, Wang J, et al. TCMSP: a database of systems pharmacology for drug discovery from herbal medicines. J Cheminform 2014;6:13.

13. Huang C, Li R, Shi W, et al. Discovery of the antitumor mechanism of calycosin against colorectal cancer by using system pharmacology approach. Med Sci Monit 2019;25:5589-93.

14. Li M, Yu L, She T, et al. Astragaloside IV attenuates Toll-like receptor 4 expression via NF- $\kappa \mathrm{B}$ pathway under high glucose condition in mesenchymal stem cells. Eur J Pharmacol 2012;696:203-9.

15. Shi G, Mao ZM, Wan YG, et al. Pathomechanisms of podocyte injury in diabetic nephropathy and interventional effects of Chinese herbal medicine. Zhongguo Zhong Yao Za Zhi 2016;41:2416-21.

16. Piñero J, Queralt-Rosinach N, Bravo À, et al. DisGeNET: a discovery platform for the dynamical exploration of human diseases and their genes. Database (Oxford) 2015;2015:bav028.

17. Lopes CT, Franz M, Kazi F, et al. Cytoscape Web: an interactive web-based network browser. Bioinformatics 2010;26:2347-8.

18. Franceschini A, Szklarczyk D, Frankild S, et al. STRING v9.1: protein-protein interaction networks, with increased coverage and integration. Nucleic Acids Res 2013;41:D808-15.

19. Subudhi AK, Boopathi PA, Pandey I, et al. Disease specific modules and hub genes for intervention strategies: a co-expression network based approach for Plasmodium falciparum clinical isolates. Infect Genet Evol 2015;35:96-108.

20. Doncheva NT, Assenov Y, Domingues FS, et al. Topological analysis and interactive visualization of biological networks and protein structures. Nat Protoc 2012;7:670-85.
21. Huang da W, Sherman BT, Lempicki RA. Systematic and integrative analysis of large gene lists using DAVID bioinformatics resources. Nat Protoc 2009;4:44-57.

22. van Dam RM, Naidoo N, Landberg R. Dietary flavonoids and the development of type 2 diabetes and cardiovascular diseases: review of recent findings. Curr Opin Lipidol 2013;24:25-33.

23. Keter LK, Mutiso PC. Ethnobotanical studies of medicinal plants used by Traditional Health Practitioners in the management of diabetes in Lower Eastern Province, Kenya. J Ethnopharmacol 2012;139:74-80.

24. Xu Y, Fisher GJ. Receptor type protein tyrosine phosphatases (RPTPs) - roles in signal transduction and human disease. J Cell Commun Signal 2012;6:125-38.

25. Malakar P, Chartarifsky L, Hija A, et al. Insulin receptor alternative splicing is regulated by insulin signaling and modulates beta cell survival. Sci Rep 2016;6:31222.

26. Stanford SM, Aleshin AE, Zhang V, et al. Diabetes reversal by inhibition of the low-molecular-weight tyrosine phosphatase. Nat Chem Biol 2017;13:624-32.

27. Benter IF, Benboubetra M, Hollins AJ, et al. Early inhibition of EGFR signaling prevents diabetes-induced up-regulation of multiple gene pathways in the mesenteric vasculature. Vascul Pharmacol 2009;51:236-45.

28. Akhtar S, Yousif MH, Dhaunsi GS, et al. Activation of ErbB2 and downstream signalling via Rho kinases and ERK1/2 contributes to diabetes-induced vascular dysfunction. PLoS One 2013;8:e67813.

29. Muhammad IF, Borné Y, Bao X, et al. Circulating HER2/ ErbB2 levels are associated with increased incidence of diabetes: a population-based cohort study. Diabetes Care 2019;42:1582-8.

30. Wang Z, Shah OJ, Hunter T. The transcriptional coactivators $\mathrm{p} / \mathrm{CIP}$ and SRC-1 control insulin resistance through IRS1 in obesity models. PLoS One 2012;7:e36961.

31. Rowan BG, Weigel NL, O'Malley BW. Phosphorylation of steroid receptor coactivator-1. Identification of the phosphorylation sites and phosphorylation through the mitogen-activated protein kinase pathway. J Biol Chem 2000;275:4475-83

32. Ren X. Effect of Fyn on hyperglycaemic induced podocytosis. Shandong University 2014. doi: 10.7666/ d.Y2596976.

33. Zhang X, Bao S, Lai D, et al. Intravitreal triamcinolone acetonide inhibits breakdown of the blood-retinal barrier through differential regulation of VEGF-A and its receptors in early diabetic rat retinas. Diabetes 
2008;57:1026-33.

34. Cai Y, Li X, Wang YS, et al. Hyperglycemia promotes vasculogenesis in choroidal neovascularization in diabetic mice by stimulating VEGF and SDF-1 expression in retinal pigment epithelial cells. Exp Eye Res 2014;123:87-96.

35. Wang CT, Wang DB, Liu KR, et al. Inducing malignant transformation of endometriosis in rats by long-term

Cite this article as: Li J, Huang Y, Zhao S, Guo Q, Zhou J, Han $\mathrm{W}, \mathrm{Xu}$ Y. Based on network pharmacology to explore the molecular mechanisms of astragalus membranaceus for treating T2 diabetes mellitus. Ann Transl Med 2019;7(22):633. doi: 10.21037/atm.2019.10.118 sustaining hyperestrogenemia and type II diabetes. Cancer Sci 2015;106:43-50.

36. Fukui M, Tanaka M, Hasegawa G, et al. Association between serum bioavailable testosterone concentration and the ratio of glycated albumin to glycated hemoglobin in men with type 2 diabetes. Diabetes Care 2008;31:397-401. 